\title{
Synthesis of enantiopure fused bisthiazolidines and thiazolidinyloxazolidines.
}

\author{
Cecilia Saiz, Valerie Castillo, Graciela Mahler \\ Cátedra de Química Farmacéutica, DQO, Facultad de Química, Gral Flores 2124, Montevideo, Uruguay \\ *gmahler@fq.edu.uy
}

Keywords: bisthiazolidines, thiazolidinyloxazolidines, fused-heterocycles.

\section{INTRODUCTION}

Among the different possible methods leading to nitrogen-containing heterocycles, iminium ion cyclization is a widely used process. Cyclic iminium ions of general structure 1 (Figure 1), bearing a nucleophilic tether with a suitable located oxygen, sulfur or nitrogen, are important building blocks for the preparation of synthetically and biologically relevant condensed heterocycles.

Figure 1. Cyclic iminium ion 1, compounds $\mathbf{2}$ and $\mathbf{3}$.

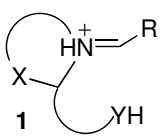

$\mathrm{X}=\mathrm{O}, \mathrm{S}, \mathrm{N}, \mathrm{C}$

$\mathrm{Y}=\mathrm{O}, \mathrm{S}, \mathrm{N}$

$\mathrm{R}=$ alkyl, aryl
The present work describes our findings in the synthesis of enantioenriched new fused thiazolidinyloxazolidines (2) and bisthiazolidines (3) via the generation of cyclic iminium ions (Figure 1).

\section{RESULTS AND DISCUSSION}

The thiazolidinyloxazolidines 2 were prepared by a 2-step sequence using a modified protocol published previously. ${ }^{2}$ Syn-bicycles 2a-d, were obtained in good yields and high enantiomeric excess, see Table 1.

Table 1. Synthesis of thiazolidinyloxazolidines 2a-d

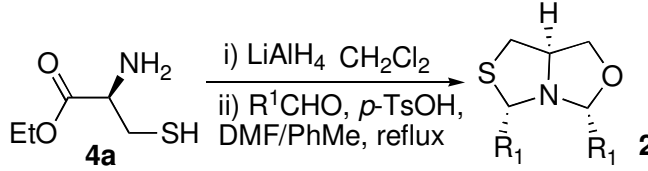

\begin{tabular}{|c|l|c|c|}
\hline Compound & \multicolumn{1}{|c|}{$\mathbf{R}^{\prime}$} & Yield (\%) & de \%' \\
\hline syn-2a & $\mathrm{Ph}$ & 89 & 99 \\
syn-2b & $p$-CIPh & 86 & 98 \\
syn-2c & $p-\mathrm{NO}_{2} \mathrm{Ph}$ & 89 & 99 \\
syn-2d & $\mathrm{CO}_{2} \mathrm{Et}$ & 60 & 98 \\
\hline
\end{tabular}

de: diasteromeric excess, determined by ' $\mathrm{H}$ NMR.

$14^{\text {th }}$ Brazilian Meeting on Organic Synthesis $-14^{\text {th }}$ BMOS - September 01-05, 2011-Brasilia, Brazil
Compounds $\mathbf{3}$ were obtained by heating $\mathbf{4 a}$ or $\mathbf{4 b}$ in the presence of the dimeric aldehyde $\mathbf{5}$, in acidic media, see Figure 2. Smooth decomposition of dithiane 5 led to the formation of 2mercaptoacetaldehyde. The reaction of two molecules of aldehyde in the presence of aminothiol $\mathbf{4 a}, \mathbf{b}$, led to the formation of fused bisthiazolidines $\mathbf{3 a}, \mathbf{c}$. Interestingly when we use $\mathbf{4 b}$ as starting material, the diastereomeric excess was higher than when we use ester $\mathbf{4 a}$, see Figure 2.

The double cyclization process led to bicycle $\mathbf{3}$ via iminium ion formation. Further studies in the serie 2 are being carried out in order to study the scope and limitations of this methodology.

Figure 2. Synthesis of fused bisthiazolidines 3

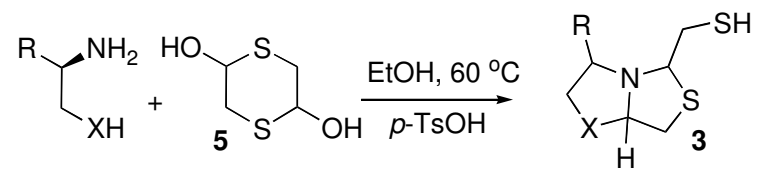

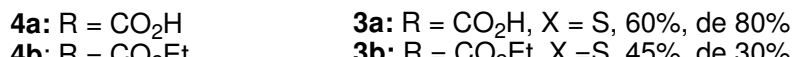

\section{CONCLUSION}

The results presented herein provide evidence for the versatility of $\mathrm{N}$ acyliminium ions for the synthesis of new bicyclic compounds not easily accessed by other routes. This structure represents new enantiomerically pure scaffolds; and we can envision their application in organocatalysis, new materials or medicinal chemistry.

\section{ACKNOWLEDGEMENTS}

V. Martínez and $\mathrm{H}$. Pezaroglo for RMN spectra. CSIC-grupos; PEDECIBA; ANII.

\section{REFERENCES}

${ }^{1}$ Royer, J.; Bonin M.; Micouin L. Chem. Rev. 2004, 104, 2311-2352.

${ }^{2}$ Saiz, C., Wipf, P., Manta, E., Mahler, S. G. Org. Lett. 2009, 11, 3170-317. 